\title{
Research on Image Color Control Based on ICC Profile
}

\author{
Jia Wang \\ Yunnan Open University, Kunming, Yunnan, 650000
}

Keywords: Image Color Control, ICC Profile, Color Management

\begin{abstract}
Color management is realized through device profile and gamut mapping. The device profile records information about the device's color characteristics and media, viewing color conditions, which is one of the elements of color management. Analysis of the gamut of different rendering intents indicates that the color gamut of perceived rendering intent does not reflect the gamut of the device because the target device is unknown when the device profile is created, so the color conversion of the perceived rendering intent is not reciprocal. Using the rendering intent media reference color gamut can regulate the perceived rendering intent gamut, which facilitates the computation of color conversions. Analysis of the gamut mapping shows that using the device connection profile results in better color conversion.
\end{abstract}

\section{Introduction}

With the advancement of computer science and the development of color input and output technology, computer graphics and image are widely used in various fields as information carriers. No matter in the printing industry, video industry, advertising industry or other color image applications, people are more and more demanding on the color quality of graphic images. For example, we hope that the TV can show a more realistic color [1]. The color of the product image and the color of the product are exactly the same when the product is purchased online. It is hoped that the captured photo can still be displayed after being transmitted through the internet or output. To meet these needs, there must be an ideal color input and output device. However, due to many factors such as imaging mechanism, component performance, machining assembly precision, consumables characteristics, and machine performance cost ratio and other factors, the "hardware" performance of the input and output devices to reproduce colors is not ideal at present and the following defects still exist. Various devices There are obvious device nonlinearities in the scanner and printer, especially the printout is affected by many factors such as the paper, the half toning algorithm, the geometric properties of the printed dots, the order of scanning and printing, the positioning accuracy of the color register, etc. The nonlinearity The relationship is complex and varies depending on the output process. The printer reproduces only part of the color, and many saturated, bright, and dark colors cannot be reproduced beyond the printer's color gamut. Due to the above "hardware" defects, the color input and print output of the color map will appear serious color cast and details of the distortion, the quality far less than the high-performance input and output requirements [2].

Founded in 1993, ICC now has more than 80 members. Its mission is to establish, develop and encourage open, hardware-independent, cross-platform color management systems and components and their standardization. ICC has studied and developed color management standards to help software developers and hardware manufacturers work together to maintain the color integrity of digital images. All input and output devices in the color flow should have their own ICC profiles that record information such as the color gamut and non-linear calibration parameters of the device in a device-independent color space so that the appropriate color management software can use these Information The color is transmitted and converted between scanners, digital cameras, color displays, proofing devices, printers and other devices to achieve a consistent color reproduction across devices [3].

According to the ICC specification, the device profile describes the device's color gamut and nonlinearity accurately. Color management components based on device characterization files can 
be accurate color conversion, color images to achieve WYSIWYG. Due to the introduction of color management software and its good compatibility, the operator is freed from a great deal of cumbersome manual color correction work by using non-standard mode [4].

\section{ICC Profile and Management System Model}

ICC color profile is mainly used for color space conversion. The ICC uses a virtual, device-independent color space, the Profile Connection Space (PCS), as the midtone space for color space conversion through the color device color space (RGB or CMYK) and the PCS space The link between the equipment for the establishment of equipment profile (Profile), enabling the color of the open management (Figure 1), making the color transfer does not depend on the color equipment. PCS space is defined by CIEXYZ space or CIELAB space. The ICC standard specifies a total of seven profile profiles, including profile profiles of three basic devices, namely, input profile files, display profile files, and output profile files; and four additional profile profiles, that is, Device Link Profile, Color Space Conversion Profile, Abstract Profile, and Named Color Profile.

Color management is a collection of applications and resources that automate the color conversion between different devices and media and maximize the fidelity of color profiles. Color management is different from traditional systems that need to be referenced by a large number of measurements. The traditional color conversion system is closed, with a single input and output, the operator need only be familiar with the experience of input and output characteristics can be well adjusted parameters to optimize system performance. With the opening up of the production environment, the input sources of graphic images are constantly increasing, and the ways of outputting are also varied. This way of working is no longer valid [5].

Closed color conversion needs to establish a color mapping between a single input and output color space, and this mapping applies only to both devices, which is a frustrating option for multi-input-multi-output color systems. The CIE-based color conversion only needs to create a color profile for each device. The mapping between the device's color space and the CIE color space can realize the color conversion between any devices. The open color management of the ICC is based on Proposed this idea, the color management model shown in Figure 3. The ICC defines the CIE XYZ and CIE L * a * b* color spaces as a color swap space (PCS) that establishes the format standard for the device color profile that records the conversion relationship between the device color space and the PCS and is used for Color management The color equipment is calibrated repeatedly and the mapping relationship between the device color space and the standard color space is established through a dedicated color feature extraction tool and stored in the corresponding color profile. The color management system, according to the color profile of the source device and the target device, the color value of the device is converted to the color value of the target device. Clearly, an open color management architecture requires not only a uniform device color profile format and device-independent color space, but also a standard framework that handles different types of color profiles and manages color conversions [6].

The profile contains the color reproduction characteristics of the color device and is the input source for the color management module. Color Management Module CMM (color management module) uses PCS as the swap color space to connect configuration files for color conversion between any device. The application calls CMM to achieve the color conversion of user's needs. Operating system color management interface, it is the link between the application and the CMM, the application of the standard color management interface calls will be converted to the corresponding CMM call. It can load different color management modules for more support with different color characteristics and management methods.

The printer's gamut space is the most complex of all color devices, and the hardest to represent. Typically, the printer color profile uses a lookup table model for data storage, so the printer color profile should include a look-up-table label (B2A) from the PCS to the Device Color Space in addition to the basic content, Different levels require the ability to add look-up tables in various copy modes and look-up table tags (A2B) from Device Color Space to PCS [7]. 


\section{ICC-Based Color Management}

The purpose of color management is to achieve consistent reproduction of color information on a variety of input and output devices. Different input / output devices have different color gamuts, adopt different color forming mechanisms, use different device-related color spaces, and have serious non-linear relationships between their respective device-related color spaces and device-independent color spaces. Therefore, color management needs to solve the problem of non-linear correction of color gamut matching and device characteristics between different devices. Since different I / O devices use different color-forming mechanisms, it is necessary to introduce device-independent color space and device-related color space to solve the above problem. In the current color management mechanism, the color gamut matching is done in the device-independent color space, and the device nonlinear correction is achieved by establishing the mapping relationship between the device-independent color space and the device-related color space. The key to the color management of the output device is how to map the color image color values of the device-independent color space to the printer color gamut through the color gamut matching and then convert the colorimetric values of the device-related color space to the colorimetric values of the device-related color space through the non-linear calibration, that is, the digital drive value is output.

ICC-based color management means that basic color management functions are implemented at the operating system level, so it is application independent. In addition, it must comply with ICC standards. (1) ICC-based color management system architecture ICC-based color management system structure shown in Figure 2. In the operating system, Color Management Frame-work is responsible for operating the most important color management functions of the system, and this mechanism provides an interface to various color management modules (CMMs). The operating system supports the default CMM features, and other CMM features can be added as long as they are as intended and available. CMM is the core of a color management system that converts color data from one device's color space to another. Color management mechanism supports CIEXYZ and CIELAB two standard color space.

ICC-based color management consists of two important stages: ICC profile generation and color matching. In Phase 1, the goal is to create an ICC profile by marking an image file to map the source device's color space to a standard midtone space, or to map the middle color space to the target device's color space. In ICC, this intermediate color space is called the profile connection space (PCS). PCS provides an unambiguous interface between the input and output profile. The important tasks in this phase are color detection and color correction. Profile is used to compensate for the color characteristics of the device, allowing the device to match the color of the original image. Today's profile generation tools have been able to provide satisfactory scanner inspection tools and calibration packages such as IT8 patches. Some vendors have provided tools for characterizing displays, such as COLORTRON. However, color characterization of printers has proved difficult due to signal-noise factors. In phase 2, the operating system uses the input and output device profiles to map the source color space data to the output color space. Here are two questions that are implied: What is the best color-space matching and how to calculate the color map? On the first issue, the ICC profile defines a tag called Rendering Intent that allows the user to choose one of the most satisfying Ways to match the source image. The ICC profile supports 4 types of rendering intents: Perceptual Matching, Relative Color Matching, Saturation Matching, and Absolute Color Matching. The second problem is pure mathematical conversion.

\section{Conclusions}

With the rapid development of computer technology, the digital color input and output technology has been rapidly developed. Color displays, scanners, digital cameras and color printers have been widely used in a wide range of applications. However, different areas require different colors, and their requirements for color equipment are as consistent as possible. In order to meet the different requirements of different fields, color equipment must have a strong color management 
capability. On this basis, ICC proposed the concept of color management. This paper introduces the appearance of color management in detail, the composition of ICC Profile, ICC color model, the overall framework, and gives the color printer ICC Profile document generation process.

\section{Acknowledgements}

Fund Project: Based on ICC profiles soft screen proofing analysis, Yunnan Provincial Science and Technology Department of applied basic research youth project, project number: 2015FD039

\section{References}

[1] Song Zhao. CIECAM02 in ICC color management system [J]. Journal of Printing Quality and Standardization. 2006 (11): 11-13.

[2] Zhang Jie, Liu Chunmiao. Scanner profile creation [J]. Printing Technology. 2009 (01),123-131.

[3] Si Lili, Liu Haoxue, Jiang Wenyan. Study on algorithm model of output device profile creation software [J]. Principle of Printing and Packaging, 2009 (03):19-21

[4] Zhang Linlin. Digital test version for ICC profiles (top) [J]. Digital Printing. 2009 (07) :62-65.

[5] Zhang Linlin. Digital test version for ICC profiles (below) [J]. Digital Printing. 2009 (09) :77-81

[6] Mo Chunjin. Color management: concept, content and its innovation and development [J]. Printed by the magazine. 2009 (12) :197-201

[7] Xu Zhaohui. How to more effectively manage color in Photoshop [J]. Printed World. 2003 (01) :18-21 